Relatively few antiprotons annihilate in flight when they are brought to rest in matter. Most annihilate only after being captured by an atom of the stopping substance. It was long thought they could not survive more than a picosecond after capture. Recent experiments with antiprotons stopped in helium are changing this picture.

\title{
How Long Do Antiprotons Last?
}

In a perfect vacuum the antiproton (p) should last exactly as long as the proton $(p)$ does - at least $10^{32}$ years according to the latest experimental limit on the proton lifetime. In the imperfect (but nevertheless very good) vacuum achieved by the HarvardMainz group at CERN which is measuring the antiproton charge-to-mass ratio to very high precision, a handful of antiprotons has been held for several months using electromagnetic fields to turn them back when they approach the walls of the vacuum chamber. In the presence of ordinary matter, the answer is much more nuanced and depends on the details of the interactions of the antiproton as it moves through the surrounding substance. It will sometimes happen that the antiproton scores a direct hit on a nucleus and comes within range of the annihilation-producing quantum chromodynamic or "strong" force. However the small size of the nucleus compared with the size of the atom makes these encounters rather infrequent in comparison with the atomic ones that excite or ionize the atom, but leave the antiproton intact. Relatively few annihilations therefore occur in flight and we shall ignore them in the following.

\section{Deceleration}

To know how long $\bar{p}$ last we must therefore start by estimating the time taken by charged particles to pass through matter and come to rest in it from a given energy. If the energy loss per unit path length $(\mathrm{d} E / \mathrm{d} x)$ is known, the deceleration time between two energies $E_{1}$ and $E_{2}$ for a velocity $v$ is:

$$
t_{\text {decel }}=\int_{E_{2}}^{E_{1}} \mathrm{~d} E(\beta c \mathrm{~d} E / \mathrm{d} x)^{-1} .
$$

where $c$ is the speed of light and $\beta=v / c$.

The Bethe-Bloch $d E / d x$ formula is part of every physicist's intellectual luggage. Its derivation assumes that the projectile interacts with atoms mainly via energy dissipating collisions with electrons, and that the impulse given to each electron is that which would be obtained if the projectile traversed the atom in a straight line. This is equivalent to assuming that the projectile is not inside the atom long enough for the atom to "know" whether its charge is negative or positive. Above about $1 \mathrm{MeV}$ (i.e., when the projectile's kinetic energy is large compared with atomic excitation or ionization energies) this is quite a reasonable assumption, and

John Eades has been an experimental physicist with CERN, CH-1211 Geneva 23, since 1970. He studied at the University of Liverpool and has worked at Columbia University, New York, USA, and at The Niels Bohr Institute in Copenhagen.

\section{J. Eades \\ CERN, Geneva, Switzerland}

Fig. $1-A$ representation of the metastable "atomcule" consisting of an antiproton $\bar{p}$, an electron $e$; and an alpha particle $\alpha$. The antiproton circular trajectory with quantum numbers $n=38, I=37$ is drawn in perspective and is to a very good approximation classical, with a frequency much lower than that of the electron, which is in the ground state (dominantly 1S). The electron's motion is not of course classical and is shown as a density distribution, polarized by the presence of the $\bar{p}$. The $\bar{p}$ velocity is so low that the electron cloud follows the antiproton around the atom. Figure courtesy of T. Nagae, INS, Tokyo.

the behaviour of protons and antiprotons at these energies is therefore very similar except for the occasional direct nuclear collisions referred to above. As $\bar{p}$ cannot be created with less than $1 \mathrm{GeV}$ of kinetic energy [1], they begin their travels well inside the Bethe-Bloch domain. Quite subtle differences in the behaviour of $p$ and $\bar{p}$ nevertheless exist at MeV energies, and new effects are continually being discovered [1]. Less subtle but better known is the repeated capture and loss of electrons, which is possible for $p$ but not for $\bar{p}$. However, we shall not make too big an error if we ignore these differences and take $t_{\text {decel }}$ for $\bar{p}$ as roughly equal to the value for protons given for $E_{2}=10 \mathrm{eV}$ in Janni's Atomic Data and Nuclear Data Tables [2]. Here the Bethe-Bloch formula is used down to $1 \mathrm{MeV}$ and various low-energy substitutes and empirical fits are adopted between $1 \mathrm{MeV}$ and $10 \mathrm{eV}$. Taking $E_{1}$ as $2 \mathrm{MeV}$ gives $t_{\text {decel }}=25.1 \mathrm{~ns}$ for hydrogen at normal temperature and pressure (NTP) and $32.4 \mathrm{~ns}$ for NTP helium. At higher pressures the deceleration times are proportionately shorter because the $\bar{p}$ spends less time travelling through the empty space between atoms.

\section{Capture}

In fact it is only when the projectile energy nears the $10 \mathrm{eV}$ cutoff of the Janni tables that the most dramatic effect of the projectile charge becomes apparent. Below a few tens of $\mathrm{eV}$, negative particles can go from continuum states to bound ones, liberating an atomic electron into the continuum as they do so. This is the normal fate of $\bar{p}$ once their energy falls below atomic excitation and ionization energies. These new atoms (as well as those formed by other negative particles) are known as exotic atoms. Once the antiproton is bound it is very difficult to

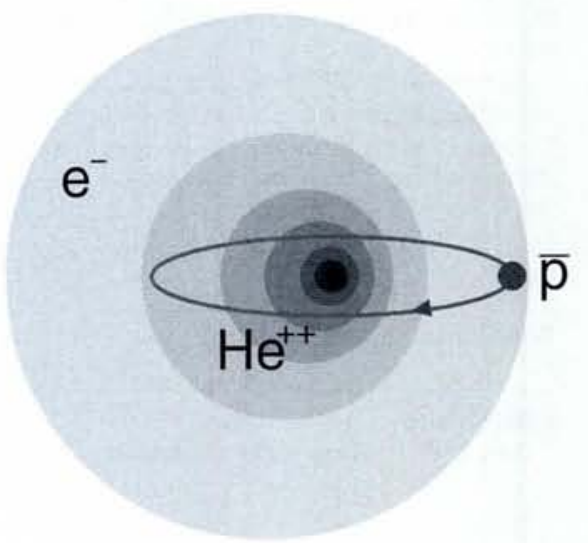

shake it loose again, and it will cascade through a series of atomic levels until it comes within range of strong interactions and annihilates. This cascade time $t_{\text {casc }}$ must be added to $t_{\text {decel }}$ to obtain the total lifetime against annihilation. Some of the general features of exotic atoms can be deduced from the formulae for the energy and radius of Bohr orbits (neglecting the interaction between electrons):

$E_{n}^{\bar{p}}=-\frac{1}{2} M(\alpha c)^{2} Z^{2} / 2 n^{2} \quad \alpha_{n}^{\bar{p}}=h n^{2} / M Z \alpha c$ $E_{n}^{e^{-}}=-\frac{1}{2} m(\alpha c)^{2} Z^{2} / 2 n^{2} \quad \alpha_{n}^{e^{-}}=\hbar n^{2} / m Z \alpha c$ Here $\alpha$ is the fine-structure constant, $Z$ the nuclear charge, $M$ the antiproton reduced mass, $m$ the electron reduced mass, and $n$ a positive integer (the principal quantum number). When the exotic atoms are formed, the atomic states that are populated are those for which the $\bar{p}$ Bohr orbit most closely overlaps that of the displaced electron. Inspection of these equations reveals a) that the $\bar{p}$ orbital energies will then be near the orbital energy of the electron, and b) that their $n$-values will be close to $\sqrt{M / n}$ (i.e., 30 for hydrogen, 38 for helium). The maximum available $\bar{p}$ kinetic energy can be taken as roughly equal to the ionization potential (13.6 eV for hydrogen, $24.6 \mathrm{eV}$ for helium), since above this value, ionization still competes strongly with capture. The maximum angular momentum brought in by the $\bar{p}$ is then the ionization potential times the Bohr radius, which just allows all possible angular momentum sub-states I (up to $I=n-1)$ to be populated. The $\bar{p}$ wavelength at these energies is about $n$ times smaller than the mean radius of the electron cloud, which implies that the $\bar{p}$ 's orbit is to a good approximation classical. In the case of helium, the $\bar{p}$ orbital velocity is smaller than that of the remaining electron by the same factor $n$. 


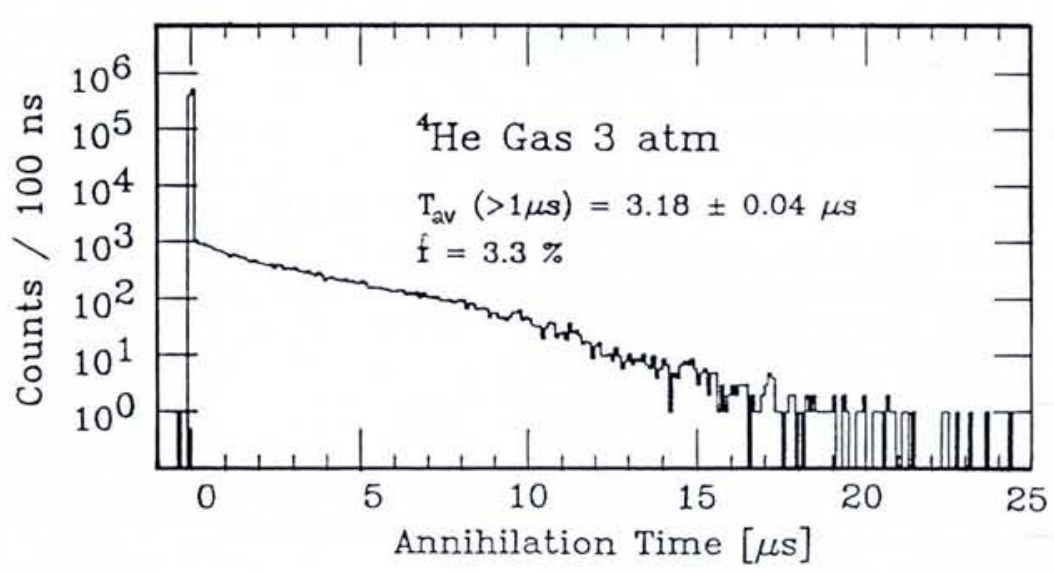

Several authors have studied the cascade theoretically and find that it is strongly influenced by collisions with neighbouring atoms, which quickly induce transitions from high- $n$, high-/ states to high- $n$ S-states (the so-called Stark mixing effect). As S-state wave functions overlap the nucleus it is easy for the antiproton to annihilate from them. In exotic atoms other than those formed in hydrogen, Auger emission also plays an important rôle in de-excitation from high to low 1 -values, and completely dominates over radiative transitions. Typically $t_{\text {casc }}$ turns out to be of order $10^{-12} \mathrm{~s}$ to $10^{-13} \mathrm{~s}$ in hydrogen or helium.

Effectively, in allowing itself to be captured by an atom, the $\bar{p}$ has only bought itself a short reprieve against annihilation. Could the $\bar{p}$ live longer by hiding from the strong interaction before capture, thereby increasing $t_{\text {decel }}$ ? It has been speculated that such hiding places may indeed exist in certain media. Antiproton analogues to hydrated electron states in water, and colour centres in crystals have been suggested as possible candidates, and the possibility has been raised that $\bar{p}$ might be trapped in superfluid $4 \mathrm{He}$ and in degenerate liquid ${ }^{3} \mathrm{He}$, and that voids or bubbles might form around $\bar{p}$ in ordinary helium. Energy loss formulae take none of these structural or physicochemical properties of the medium into account, so it would not be entirely surprising to find larger values for $t_{\text {decel }}$ in certain

\section{LEAR}

The Low Energy Antiproton Ring (LEAR) has produced high quality, low energy antiproton beams since 1984 by taking particles from CERN's antiproton storage complex at $3.5 \mathrm{GeV} / c$, decelerating them in a ring of magnets, and cooling away the unavoidable (Liouville theorem) phase space blow-up. Beams of $2 \mathrm{MeV}$ with a sharply defined energy and $2 \mathrm{~mm}^{2}$ cross-sectional area are routinely available. The antiprotons are are produced by bombarding a target with $26 \mathrm{GeV}$ beams from the Proton Synchrotron. From the resulting spray of secondary particles, a focussing system guides antiprotons into the Antiproton Accumulator and Collector comprising two concentric rings that collect, $\mathrm{cool}$ and store particles for injection into LEAR.

Fig. 2 - The time-interval spectrum between entry of $\bar{p}$ into a ${ }^{4} \mathrm{He}$ target at 3 atmospheres pressure and its annihilation. The fraction $f$ of the spectrum outside the prompt annihilation peak is $3.3 \%$, and the average time interval $T_{a v}$ is evaluated from a starting value of $1 \mu \mathrm{s}$ after the immediate annihilation peak at the left.

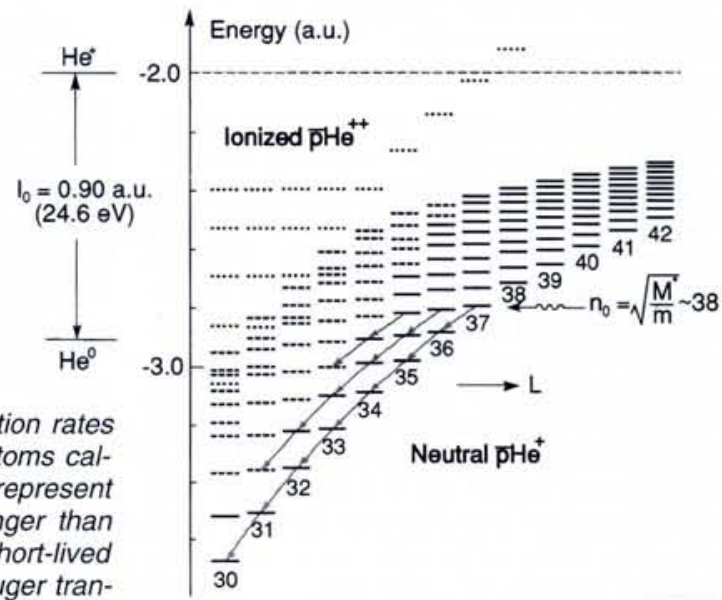

Fig. 3- Level structure and transition rates of metastable antiprotonic helium atoms calculated by $K$. Ohtsuki. Solid lines represent metastable states with lifetimes longer than $2.5 \mathrm{~ns}$; broken lines represent short-lived states of this atom dominated by Auger transitions which remove the second electron.

The dot-dash lines are ionized $\bar{p} \mathrm{He}^{++}$states (i.e., with the second electron ejected). The main stream of slow radiative transitions is indicated by arrows.

special media. So far however, none of the above-mentioned hiding places has been found, although it is true to say that no careful search has ever been made.

\section{A New Phenomenon}

In 1991 this picture changed dramatically when it was found by Iwasaki et al. [3] working at KEK - the national high-energy physics laboratory in Japan - that the annihilation of some of the $\bar{p}$ stopped in liquid helium was delayed by up to $15 \mu$ s after the beam entered the helium target. No such effect has been seen in protonium (the exotic hydrogen atom consisting of a $p$ bound to a $\bar{p}$ ). These experiments are being continued at the LEAR machine at CERN by a collaboration of groups from Tokyo, Munich, CERN, Budapest, and Okazaki. Working in conjunction with CERN's $\bar{p}$ accumulator, LEAR decelerates antiprotons (see insert) to provide very pure and high-quality $\bar{p}$ beams with a sharply defined energy down to a few MeV and a few $\mathrm{mm}^{2}$ cross-sectional area, which allow among other things the stopping of $\bar{p}$ in gaseous targets. A typical annihilation-time spectrum in gas is shown in Fig. 2 [4]. The $\bar{p}$ enter the 3 atmospheres gas target at about $2 \mathrm{MeV}$ and the histogram shows that while nearly all of them slow down, are captured and annihilate in less than $100 \mathrm{~ns}$, some $3.3 \%$ survive for times as long as $20 \mu \mathrm{s}$. This may not seem very long, but it is in conflict with the estimated $t_{\text {casc }}$ by a factor of $10^{7}$ and with the estimated $t_{\text {decel }}$ for 3 atmospheres by a factor of almost $10^{4}$. On the other hand, no effect was seen in lithium, nor in any noble gas other than helium. What was observed was an isotope effect, the mean lifetime being about $14 \%$ shorter in ${ }^{3} \mathrm{He}$ than in ${ }^{4} \mathrm{He}$.

The effect of foreign gases on the time spectrum has also been investigated and revealed strong quenching effects by hydrogen at concentrations as low as a few tens of $\mathrm{ppm}$. On the other hand gases such as xenon, krypton, argon, and neon could be added up to the percent level without much effect on the time spectrum. Various physico-chemical conditions of helium (superfluid $\mathrm{He}$, solid $\mathrm{He}$ ) were also investigated. Even in the solid state the metastability does not seem to be quenched appreciably by the numerous interatomic collisions. Many subtle differences and similarities in these spectra still await adequate interpretation, and the conclusion of a workshop held in February at the foot of Mount Fuji in Japan was that new aspects of the phenomenon need urgent study.

It is not possible to deduce from Fig. 2 whether the additional delay in the late annihilations accumulates before or after capture, but there are some good theoretical reasons to think that it may come after. Condo [5] had suggested in the 1960's that in some of the exotic high-I, high- $n$ helium atom states formed by pions and kaons, the Auger transition which ejects the second electron could be strongly suppressed, essentially because a large quantum jump in $I$ is necessary from these states to release the $24.6 \mathrm{eV}$ of electron binding energy. Protected during collisions by the charge cloud of this electron, the exotic atom would then not be able to reach S-states quickly by Stark mixing. In this case, the atom could only de-excite by radiative transitions. The rate of these depends on $n^{-3}$, so they are very slow. Russell [6] later extended this idea to $\bar{p}$ exotic atoms. His detailed calculations predicted that some circular or nearly circular Bohr orbits at high $n$ and $I$ would show this metastable behaviour. The Condo-Russell model has been developed by Ahlrichs et al. [7] and more recently by Shimamura, by Yamazaki and Ohtsuki, and by Greenland and Thürwächter [8]. The level scheme of Fig. 3 and the diagram of the $\bar{p} \alpha e^{-}$atom on the front cover and Fig. 1 are based on the results of several of these authors. Looking at this last diagram and remembering the classical, low frequency nature of the $\bar{p}$ large-/ orbits and the conse- 